\title{
Measurement of Cross-Sectional Dimensions of the Cricoid Cartilage: A Computed Tomographic Study
}

\author{
Hyun-Woo Shin, MD; Youngjin Ahn, MD; Myung-Whun Sung, MD, PhD; \\ Kwang Hyun Kim, MD, PhD; Tack-Kyun Kwon, MD, PhD
}

\begin{abstract}
Objectives: We measured the cross-sectional dimensions of the cricoid cartilage and degrees of ossification of the marrow space for designing a cricoid implant.

Methods: We reviewed 100 age- and sex-matched neck computed tomography scans that were performed at Seoul National University Hospital from 2001 to 2005. Under a standardized computed tomography window setting, the height of the cricoid cartilage marrow and the thickness of the marrow space and the ossified inner and outer cortices were measured by one observer.

Results: The mean height of the cricoid cartilage marrow was $13.6 \mathrm{~mm}$ (range, 5.5 to $20.5 \mathrm{~mm}$ ) in women and $17.5 \mathrm{~mm}$ (range, 13.0 to $24.5 \mathrm{~mm}$ ) in men. The mean thickness of the cricoid cartilage marrow was $3.17 \mathrm{~mm}$ (range, 1.22 to 4.75 $\mathrm{mm}$ ) in women and $5.13 \mathrm{~mm}$ (range, 3.42 to $7.60 \mathrm{~mm}$ ) in men. We also observed that women in general have a higher density of the cricoid marrow than men; 4 women and 1 man had a markedly denser marrow space. There were individual variations in size and density of the cricoid marrow.
\end{abstract}

Conclusions: Human adult cricoid cartilages have sufficient marrow space for cricoid implantation. Preoperative evaluation of the size and density of the cricoid marrow is recommended for individual application of the cricoid implant.

Key Words: cricoid cartilage, cross-sectional anatomy, implant, vocal fold paralysis.

\section{INTRODUCTION}

Various surgical methods have been developed for the treatment of unilateral vocal fold paralysis (UVFP), including laryngeal framework surgery, reinnervation surgery, and injection laryngoplasty. With the introduction of new injection materials such as hyaluronic acid and hydroxyapatite, injection laryngoplasty is gaining popularity as a noninvasive and effective procedure. However, conventional arytenoid adduction remains the only surgical option for patients with a large posterior glottal gap.

Considering this limitation, we introduced a novel surgical technique that is less invasive and simpler than the conventional arytenoid adduction, and we demonstrated its efficacy using an animal model. ${ }^{2}$ In that study, we used canine larynges. A nail-shaped stainless steel rod and an insertion device were designed for an in vivo trial. After unilateral recurrent laryngeal denervation surgery in 5 adult mongrel dogs, the implants were inserted endoscopically through a small mucosal incision over the cricoarytenoid joint. Acoustic and aerodynamic data were obtained from each animal before serial euthanasia followed by examination of the excised larynges. The canine cricoid cartilage demonstrated adequate marrow space for implantation. We found that the arytenoid cartilage was successfully medialized and tightly fixed over a sufficient period of time just by inserting an implant in the cricoid cartilage. The animal study showed that the implantation procedure was relatively easy and relatively safe. Acoustic and aerodynamic studies confirmed the functional improvement of the voice.

On the basis of these results, we postulated that endoscopic insertion of a cricoid implant into the marrow of the human cricoid cartilage could achieve outcomes comparable to those of conventional arytenoid adduction without cervical incision. ${ }^{2}$ To apply this technique in humans, measuring the dimensions of the cricoid cartilage and the marrow space is essential. To date, however, only a limited number of studies have investigated the exterior dimensions of the cricoid cartilage..$^{3-6}$ In particular,

From the Department of Otorhinolaryngology-Head and Neck Surgery, Seoul National University College of Medicine, Seoul, Korea. Supported by grant 03-2008-001-0 from the Seoul National University Hospital Research Fund.

Presented at the meeting of the American Laryngological Association, Orlando, Florida, May 1-2, 2008.

Correspondence: Tack-Kyun Kwon, MD, PhD, Dept of Otorhinolaryngology-Head and Neck Surgery, Seoul National University College of Medicine, 101 Daehangno, Jongno-gu, Seoúl 110-744, Korea. 


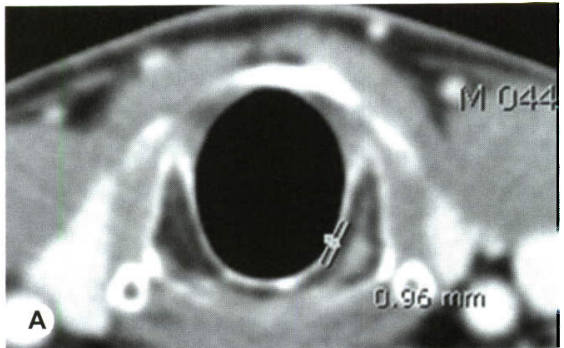

Fig 1. Thicknesses of A) inner cortex, B) outer cortex, and C) marrow space were measured on thickest portion of cricoid cartilage by means of axial computed tomographic (CT) scans.

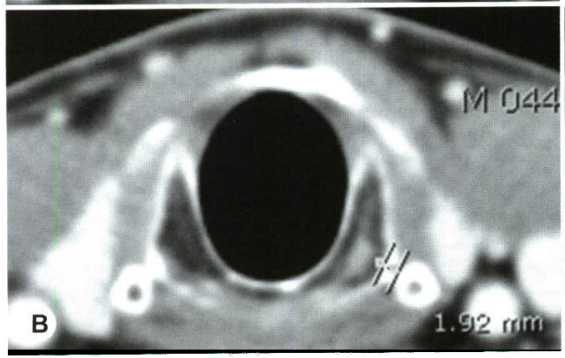

the dimensions of the cricoid marrow space had yet to be reported. For the design of the cricoid implant, we determined the anatomic aspects of the human cricoid cartilage by measuring its dimension and density from computed tomographic (CT) images.

\section{MATERIALS AND METHODS}

This study was approved by the Institutional Review Board at Seoul National University Hospital. A total of 652 neck CT scans were performed at this hospital from 2001 through 2005. Excluding patients with lesions involving the cricoid cartilage, we analyzed 100 randomly selected CT scans from male and female patients, 10 from each decade of life (age range, 20 to 69 years; mean, 45.7 years). All CT images were taken with a tube voltage of $140 \mathrm{kV}$, a tube current of $200 \mathrm{~mA}$, and a scan thickness of $2.5 \mathrm{~mm}$ and were displayed at $512 \times 512$ pixel resolution. In each image, the following were measured by one investigator using a ruler tool on the radiologic image viewer (m-view 5.4, Marotech, Seoul, Korea): the height of the cricoid cartilage and the thickness of the marrow space and the inner cortex and outer cortex of the cricoid cartilage. All distances were determined on the axis perpendicular to the tangent line of the thickest portion of the cricoid cartilage (Fig 1). According to the degree of attenuation on the CT images, the density of the marrow space was classified by one investigator into 4

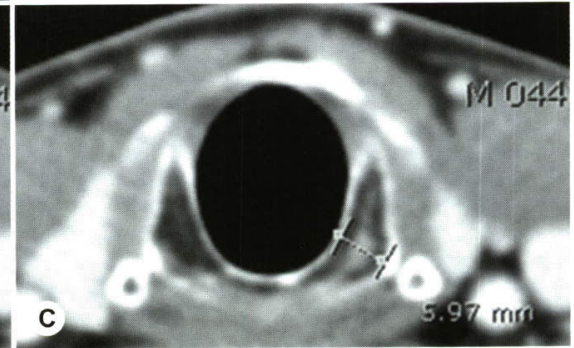

groups of minimal, low, intermediate, and high signal intensities (Fig 2). The window width, level, and zooming power of the CT images were kept constant during the measurements.

To validate the measurements based on the 2-dimensional axial CT images, we compared the size of a cricoid cartilage between CT sections and its corresponding laryngectomy specimen. The thicknesses of the cricoid cartilage in the center of the trachea and at the mid-level of the cricoid cartilage were measured under identical conditions (Fig 3). The thickness of the inner cortex, including the soft tissue lining the tracheal lumen, was $2.45 \mathrm{~mm}$ on axial CT sections and $2.16 \mathrm{~mm}$ on gross examination of the cricoid specimen. The thicknesses of the marrow space and the outer cortex were also measured as $2.04 \mathrm{~mm}$ and $0.8 \mathrm{~mm}$ on CT imaging, respectively. In addition, similar outcomes were obtained for the pathologic section of the same portion (Fig 3).

A pilot study that consisted of randomly selected CT scans from male and female patients from each decade of life (age range, 10 to 69 years; $n=60$ ) was conducted in advance to investigate whether any differences existed between the left and right sides of the cricoid cartilages. Because it revealed no significant difference between the sides regarding the thickness and density of the cricoid cartilag- 

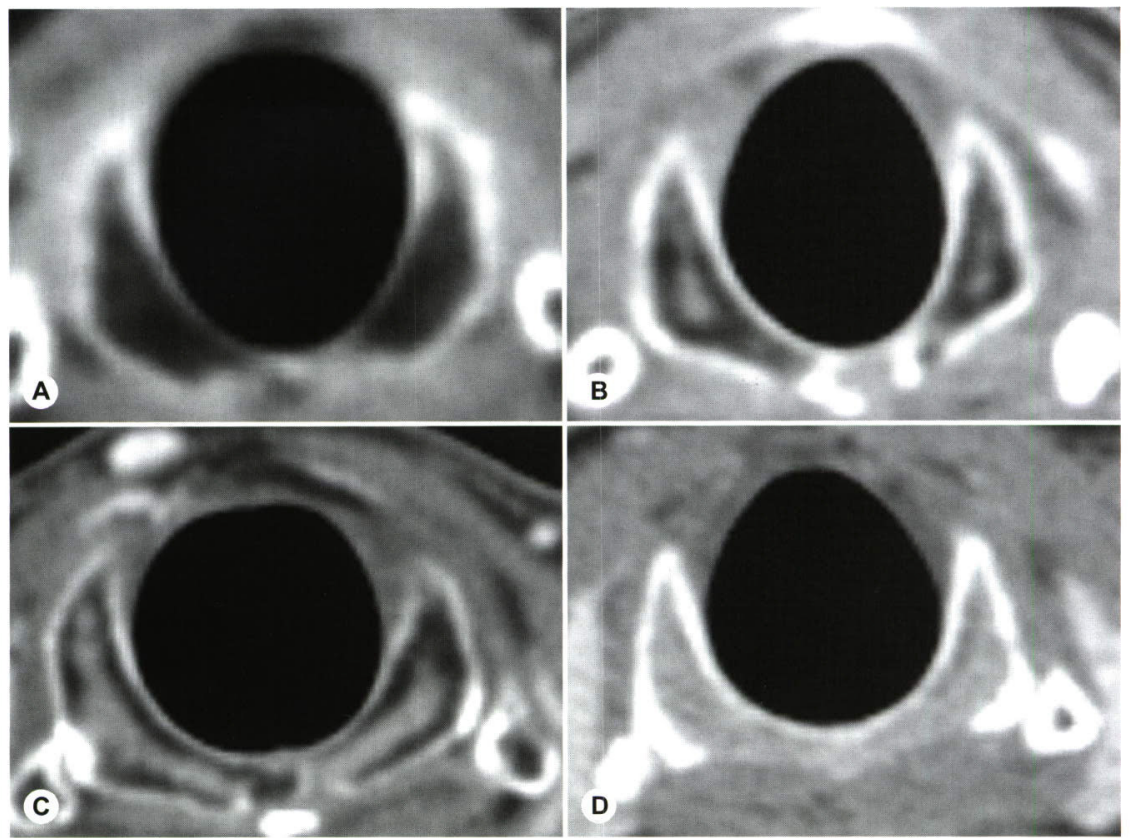

Fig 2. Density of marrow space was classified into 4 groups according to attenuation on CT images. A) Minimal intensity, same as fatty tissues (grade 1). B) Soft tissue density less than one third thickness of marrow space (grade 2). C) Soft tissue density less than two thirds thickness of marrow space (grade 3). D) Diffuse ossified marrow (grade 4).

es ( $\mathrm{p}=0.176$, paired $t$-test), we performed the measurements only on the left side. It was also found that the measurements of cross-sectional dimensions on the CT images were unreliable in patients under 20 years old, because the cortex of their cricoid cartilages lacked ossification.
Statistical analysis was performed with the statistical software package SigmaStat for Windows SPSS 12.0 (SPSS, Chicago, Illinois). Linear regression was used to analyze correlation between parameters, and a linear-by-linear association of the $\chi^{2}$ test was adopted to analyze relative patterns among

Fig 3. Comparison of measurements on $\mathbf{A}$ ) axial $\mathrm{CT}$ section and B) pathologic section $(H \& E$, original $\times 2.5$ ).
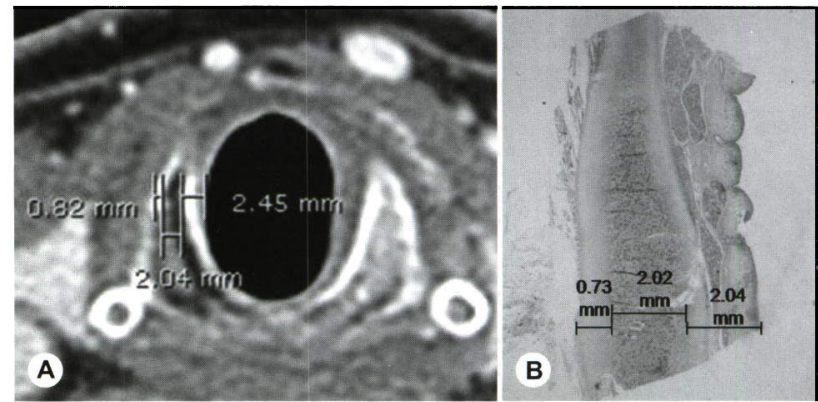
TABLE 1. THICKNESS, HEIGHT, AND DENSITY OF CRICOID CARTILAGE

\begin{tabular}{ccccccccc}
\hline & \multicolumn{3}{c}{ Men $(N=50)$} & & \multicolumn{3}{c}{ Women $(N=50)$} \\
\cline { 2 - 3 } \cline { 6 - 8 } & Range & Mean & $S D$ & & Range & Mean & $S D$ \\
\hline Thickness (mm) & & & & & & & \\
Inner cortex & $0.63-3.81$ & 2.02 & 0.95 & & $0.47-5.96$ & 2.37 & 1.14 \\
Marrow & $3.38-7.60$ & 5.13 & 0.92 & & $1.22-4.75$ & 3.17 & 1.01 \\
Outer cortex & $1.07-3.81$ & 2.36 & 0.75 & & $0.99-5.96$ & 2.47 & 1.00 \\
Height (mm) & $15.0-27.5$ & 20.5 & 3.08 & & $7.5-22.5$ & 14.6 & 3.62 \\
Density of & $1-4$ & 1.96 & 0.77 & & $1-4$ & 2.44 & 0.85 \\
marrow (grade) & & & & & & \\
\hline
\end{tabular}

discontinuous variables. A p value of less than 0.05 was considered to be statistically significant.

\section{RESULTS}

A summary of measurements is shown in Tables 1 and 2 . The mean thickness of the cricoid marrow was $3.17 \pm 1.01 \mathrm{~mm}$ (range, 0 to $4.75 \mathrm{~mm}$ ) for women and $5.13 \pm 0.92 \mathrm{~mm}$ (range, 3.42 to $7.60 \mathrm{~mm}$ ) for men. The marrow space expanded with age ( $p$ $=0.015$ in women and p $<0.001$ in men; Fig 4). In contrast, no age difference was observed in the thickness of the inner and outer cortices $(p=0.232$ and $p=0.287$ in women, and $p=0.367$ and $p=$ 0.944 in men, respectively; Fig 4).

The numbers of patients who had minimal, low, intermediate, or high signal intensity of the marrow were $23,39,33$, and 5 , respectively. The density of the marrow decreased with age $(\mathrm{p}=0.022$ in women and $\mathrm{p}=0.049$ in men), and its mean grade was higher in women in all age groups. Five cases had grade 4 density, and 4 of them were female.

The height and body mass index (BMI) of the subjects showed a positive correlation with the height of the cricoid cartilage and a negative correlation with the density of the marrow (Table 3). Specifically, the thickness of the cricoid marrow increased with the height or BMI of the subject, whereas the thickness of the inner and outer cortices showed no change. As the height or BMI of the subject increased, the thickness of the cricoid marrow increased without the increment of inner and outer cortex thickness.

\section{DISCUSSION}

In this study we found that cricoid cortical ossification appeared in the early third decade of life and progressed with aging. Whereas the space of the cricoid marrow increased with age, the density of the marrow decreased. One interesting finding was that the cricoid marrows of women were denser than those of men. This difference was probably due to the relatively narrow marrow space observed in women.

For successful endoscopic implantation, several conditions should be satisfied. First, it is important to evaluate the space necessary for cricoid implantation. Severely calcified marrow of the cricoid cartilage can hinder advancement of the implant or make the implant head in the wrong direction, ie, into the airway or the surrounding major vessels. Accordingly, it is important to know the degree of ossification of the cricoid marrow before operating. Only 5 patients ( 4 women and 1 man) had extremely calcified marrows, and most patients had sufficient marrow space for cricoid implantation. Although the calcification of the cricoid marrow around the point of insertion is not common, clinicians should recognize this finding in advance.

The implant inserted into the cricoid marrow should be fixed tightly and incapable of escape. Fortunately, the thickness of the inner cortex is about

TABLE 2. DIMENSIONS OF CRICOID CARTILAGE ACCORDING TO AGE AND GENDER

\begin{tabular}{|c|c|c|c|c|c|c|}
\hline \multirow[b]{2}{*}{ Age $(y)$} & \multirow[b]{2}{*}{$N$} & \multirow{2}{*}{$\begin{array}{c}\text { Body } \\
\text { Height }(\mathrm{cm})\end{array}$} & \multirow{2}{*}{$\begin{array}{c}\text { Height of } \\
\text { Cricoid } \\
\text { Cartilage }(\mathrm{mm})\end{array}$} & \multicolumn{3}{|c|}{ Thickness ( $\mathrm{mm}$ ) } \\
\hline & & & & Inner Cortex & Marrow & Outer Cortex \\
\hline \multicolumn{7}{|l|}{ Women } \\
\hline $20-29$ & 10 & $162.1 \pm 5.6$ & $11.5 \pm 3.2$ & $1.9 \pm 1.0$ & $2.1 \pm 1.0$ & $2.1 \pm 0.8$ \\
\hline $30-39$ & 10 & $161.1 \pm 3.5$ & $15.8 \pm 3.3$ & $2.3 \pm 1.3$ & $3.1 \pm 0.7$ & $2.5 \pm 1.0$ \\
\hline $40-49$ & 10 & $157.8 \pm 2.8$ & $14.7 \pm 3.2$ & $2.7 \pm 0.8$ & $3.8 \pm 0.6$ & $2.7 \pm 0.8$ \\
\hline $50-59$ & 10 & $156.4 \pm 2.8$ & $15.2 \pm 3.6$ & $2.5 \pm 1.1$ & $3.9 \pm 0.6$ & $2.6 \pm 0.9$ \\
\hline $60-69$ & 10 & $152.3 \pm 5.2$ & $15.8 \pm 3.7$ & $2.4 \pm 1.5$ & $3.0 \pm 1.1$ & $2.5 \pm 1.5$ \\
\hline \multicolumn{7}{|l|}{ Men } \\
\hline $20-29$ & 10 & $173.3 \pm 3.6$ & $18.8 \pm 2.1$ & $2.5 \pm 0.9$ & $4.2 \pm 0.7$ & $2.5 \pm 0.8$ \\
\hline $30-39$ & 10 & $175.4 \pm 4.9$ & $19.3 \pm 3.1$ & $1.9 \pm 1.0$ & $5.1 \pm 0.9$ & $2.3 \pm 0.7$ \\
\hline $40-49$ & 10 & $170.9 \pm 3.3$ & $20.0 \pm 2.4$ & $1.7 \pm 0.8$ & $5.3 \pm 0.6$ & $2.0 \pm 0.7$ \\
\hline $50-59$ & 10 & $167.0 \pm 5.1$ & $22.5 \pm 2.6$ & $2.2 \pm 1.2$ & $5.2 \pm 0.7$ & $2.7 \pm 0.9$ \\
\hline $60-69$ & 10 & $166.6 \pm 4.7$ & $22.0 \pm 3.7$ & $1.8 \pm 0.9$ & $5.9 \pm 1.0$ & $2.3 \pm 0.7$ \\
\hline
\end{tabular}




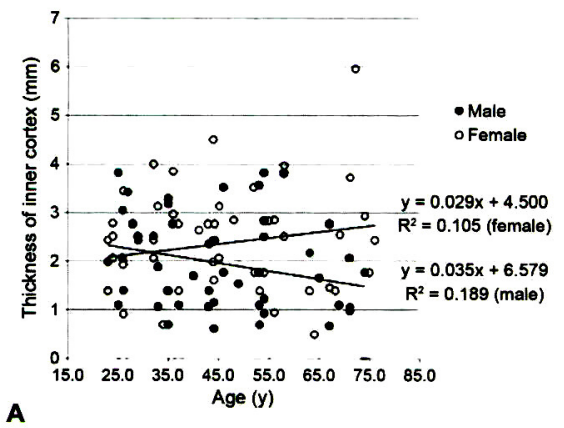

Fig 4. Correlation between age and each primary parameter of cricoid cartilage, ie, thicknesses of A) inner cortex, B) marrow space, and C) outer cortex. Linear regression was used.

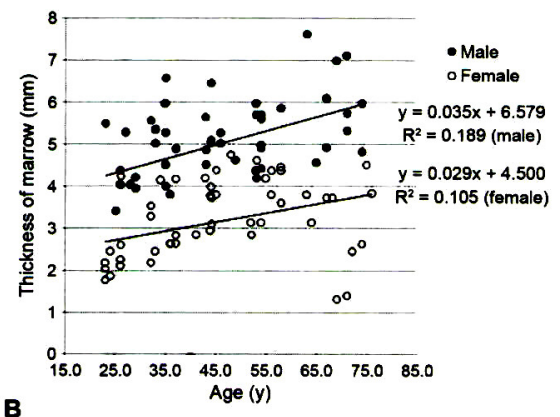

$2 \mathrm{~mm}$ and is maintained for life in most cases. This may provide the necessary protection from implant protrusion into the airway. However, 12 cases had a thickness of the inner cortex of less than $1.0 \mathrm{~mm}$, and ossification of the inner cortex was not found in 3 patients. Therefore, attention should be paid in these patients, although the tough perichondrium might help to keep the implant in the same position.

All of the cross sections were piled up vertically for reconstruction of the cricoid cartilages in men and women (Fig 5). The dimensions of the cricoid marrow were estimated to be about 18 and $14 \mathrm{~mm}$ high and 5 and $3 \mathrm{~mm}$ wide in men and women, respectively.

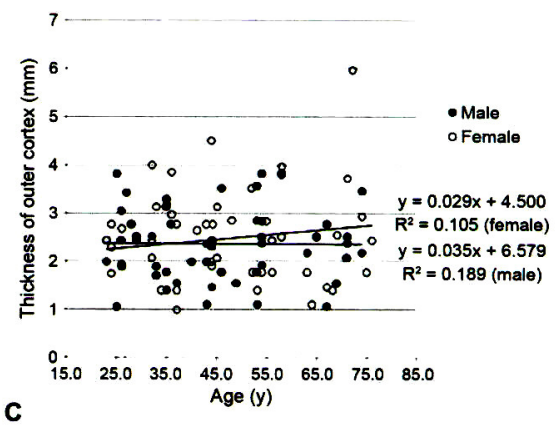

To acquire the normative values, data extracted from human laryngectomy specimens should be more accurate than those from CT images. However, because of the paucity of total laryngectomy cases and age-related occurrence of laryngeal cancers, gathering a sufficient number of specimens for analysis proves challenging. In addition, the rapidly developing CT technology can provide representative anatomic information if a consistent protocol is maintained. ${ }^{7}$ Considering that a preoperative CT scan would be performed for surgical planning in the practical setting, CT-based analysis is more useful.

Although the recent protocols of CT scanning

TABLE 3. CORRELATION BETWEEN PRIMARY PARAMETERS OF CRICOID CARTILAGE AND BODY HEIGHT OR BODY MASS INDEX

\begin{tabular}{|c|c|c|c|c|c|c|c|c|c|c|}
\hline & \multicolumn{2}{|c|}{ Height } & \multicolumn{2}{|c|}{ Density* } & \multicolumn{2}{|c|}{ Inner Cortex } & \multicolumn{2}{|c|}{ Marrow } & \multicolumn{2}{|c|}{ Outer Cortex } \\
\hline & $R^{2}$ & $p$ & $\chi^{2}$ & $p$ & $R^{2}$ & $p$ & $R^{2}$ & $p$ & $R^{2}$ & $p$ \\
\hline Body height & 0.167 & $<0.001 \uparrow$ & 6.746 & $0.009 \dagger$ & 0.020 & 0.228 & 0.251 & $<0.001 \dagger$ & 0.005 & 0.533 \\
\hline Body mass index & 0.103 & $0.005 \dagger$ & 9.621 & $0.002 \uparrow$ & 0.002 & 0.695 & 0.131 & $0.002 \div$ & $<0.001$ & 0.928 \\
\hline
\end{tabular}




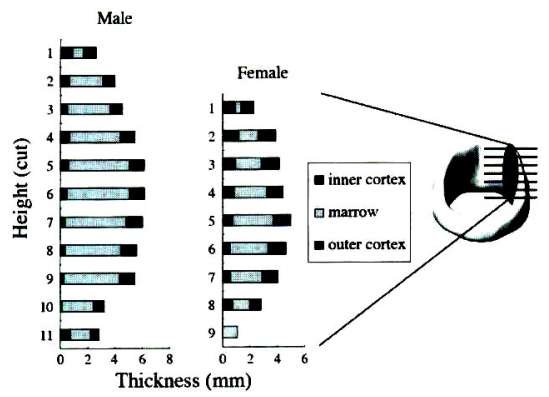

Fig 5. Schematic diagram shows contour of cricoid cartilage, including marrow space, by piling up all sectional measurements. Height is given as number of sections with $2.5-\mathrm{mm}$ thickness.

have improved considerably, the partial volume averaging effect has not been completely resolved. To date, numerous reports have cited the limitations of clinical CT scans in assessing the cortical thickness and density. ${ }^{8-11}$ Because all of the images were displayed with a pixel resolution of $512 \times 512$ and the scanned area was around $30 \times 30 \mathrm{~cm}$, the spatial resolution was calculated to be $0.6 \mathrm{~mm}$. The acrossplane resolution was the same as the thickness of the section $(2.5 \mathrm{~mm})$. These technical limitations should be taken into account when we assess the density or thickness of the cricoid cartilage. Because the cricoid cartilage lies nearly perpendicular to the axial plane of CT scans, the obscurity in the margin of the axial plane could be substantially minimized.

\section{CONCLUSIONS}

Human adult cricoid cartilages have sufficient marrow space for implantation. The estimated size of the cricoid marrow should be about $18 \mathrm{~mm}$ high and $5 \mathrm{~mm}$ wide in men and $14 \mathrm{~mm}$ high and $3 \mathrm{~mm}$ wide in women. An individual application of the cricoid implant considering the size and density of the cricoid marrow is recommended.

Acknowledgment: The authors thank Charles Tong, MS, for his assistance in proofreading this article.

\section{REFERENCES}

1. Karpenko AN, Dworkin JP, Meleca RJ, Stachler RJ. Cymetra injection for unilateral vocal fold paralysis. Ann Otol Rhinol Laryngol 2003;112:927-34.

2. Kwon TK, Jeong WJ, Sung MW, Kim KH. Development of endoscopic arytenoid adduction using cricoid implant. Ann Otol Rhinol Laryngol 2007;116:770-8.

3. Randestad A, Lindholm CE, Fabian P. Dimensions of the cricoid cartilage and the trachea. Laryngoscope 2000;110:195761.

4. Eckel HE, Sittel C, Zorowka P, Jerke A. Dimensions of the laryngeal framework in adults. Surg Radiol Anat 1994;16:31-6.

5. Eckel HE, Sittel C. Morphometry of the larynx in horizontal sections. Am J Otolaryngol 1995;16:40-8.

6. Ajmani ML. A metrical study of the laryngeal skeleton in adult Nigerians. J Anat 1990;171:187-91.

7. Yumoto E, Sanuki T, Hyodo M. Three-dimensional endo- scopic images of vocal fold paralysis by computed tomography. Arch Otolaryngol Head Neck Surg 1999;125:883-90.

8. Dougherty G, Newman D. Measurement of thickness and density of thin structures by computed tomography: a simulation study. Med Phys 1999;26:1341-8.

9. Newman DL, Dougherty G, al Obaid A, al Hajrasy $\mathbf{H}$. Limitations of clinical CT in assessing cortical thickness and density. Phys Med Biol 1998;43:619-26.

10. Prevrhal S, Engelke K, Kalender WA. Accuracy limits for the determination of cortical width and density: the influence of object size and CT imaging parameters. Phys Med Biol 1999;44:751-64.

11. Prevrhal S, Fox JC, Shepherd JA, Genant HK. Accuracy of CT-based thickness measurement of thin structures: modeling of limited spatial resolution in all three dimensions. Med Phys 2003;30:1-8. 